\title{
COMMENT
}

\section{The contributions of genetics to premature birth}

\author{
David K. Stevenson ${ }^{1}$, Ronald J. Wong ${ }^{1}$, Gary M. Shaw ${ }^{1}$, Jingjing Li $^{1}$, Paul H. Wise ${ }^{1}$ and Jonathan M. Davis ${ }^{2}$ \\ Pediatric Research (2019) 85:416-417; https://doi.org/10.1038/s41390-019-0292-0
}

The human condition has many determinants. Although some of the most important are due to genetics, their fundamental contributions to various phenotypes are only apparent through gene expression. This is in large part determined by geneenvironment interactions, some being ancestral and others occurring throughout the life-course. Such gene-environment interactions involve natural selection forces, resulting in inherited as well as epigenetic changes, which can also lead to intergenerational effects. Moreover, many human conditions, recognized as pathologic, chronic in nature, or evolving with age are polygenic in origin. They are determined not by a single gene (or a small number of genes), but by many genes collectively, imposing a biologic capacity (or incapacity) on an individual challenged with a particular environmental circumstance. Hallman and colleagues ${ }^{1}$ have provided a thoughtful overview of the current state of knowledge related to genomics and spontaneous preterm birth (SPTB) and have suggested that $25-40 \%$ may be hereditary. Moreover, they suggest that the maternal genome likely has more influence than the fetal genome on the risk of SPTB-but much remains unknown about how suspect genes identified by present genomic research might contribute to SPTB through various functional gene pathways. Nonetheless, they provide examples of candidate genes derived from recent findings, ${ }^{2,3}$ including selenocysteine-specific elongation factor (EEFSEC), WNT4, and HSPA1L, and suggest plausible gene pathways that may be relevant to defining the "clocks" that influence the risk of SPTB.

Hallman and colleagues ${ }^{1}$ also list factors associated with the onset of SPTB, including uterine distention, preterm prelabor (premature) rupture of membranes (PPROM), infectionactivated inflammation, loss of immune tolerance, intrauterine bleeding, placental and fetal growth, fetal maturity, and the endocrine system. They suggest that current pharmacological approaches to prevent or delay SPTB using antibiotics, probiotics, tocolytic agents, or progesterone have had little impact on SPTB despite evidence supporting the contributions of each of these factors. This legacy of preventive failures sets the stage for the main focus of their review, which is on the genetic predisposition to SPTB as identified by genome-wide association studies (GWAS) or other genomic approaches. More importantly, they argue that molecular and translational studies are necessary to understand the functions of the identified genes that meet significance, as well as the gene pathways that might contribute. Ultimately, it is gene expression that is important. Thus, more information about the epigenome (the methylome) and processes involved with DNA replication and transcription or translation is needed. Other "omic" measures are also important for understanding such post-transcriptional and -translational events. Although many tools have been useful in identifying candidate genes (e.g., genome-wide linkage, whole exome sequencing), GWAS have been the predominant tool used. Nonetheless, the authors conclude that at least one $\mathrm{GWAS}^{3}$ was sufficiently large for its intended purpose. This is the study that identified EEFSEC, WNT4 and a few others as genes that might have relevance to SPTB. One concern with this approach is that the proximity of a single nucleotide polymorphism (SNP) to a particular gene does not necessarily mean that the mutation will affect a change in the transcription of that gene-the closest gene is not necessarily the one that is being regulated. This is especially true in the case of enhancers, which may be important for the regulation of genes at a distance from the identified SNP. Thus, the findings by Zhang and colleagues ${ }^{2}$ should not be considered definitive, but instead should be considered suggestive of genes that may or may not be involved in SPTB.

The authors appropriately point out the importance of scale when performing a GWAS. However, even in the study by Zhang and colleagues, ${ }^{2}$ who looked at more than 40,000 study subjects, only four significant loci were identified with a risk odds ratio below 1.2. Considering that the heritability of SPTB might be between 25 to $40 \%,{ }^{1}$ the GWAS far from captures the genetic bases of SPTB, and the major genetic component is still unknown. A GWAS subscribes to the notion of "common disease, common variants," but does not begin to answer the question of whether common variants indeed constitute the molecular basis of SPTB. Increasing evidence today suggests a major role for rare variants in common genetic diseases. The aggregation of rare variants, each with high penetrance, is the major contributor to the genetic risk of complex diseases. ${ }^{4}$ Therefore, to capture the missing heritability in SPTB, future studies are warranted to examine the role of rare variants, which can only be revealed by sequencing technologies.

Although the mutations discussed so far are all germ-line mutations, mutations could also occur de novo and are more likely to be associated with severe medical conditions. Hallman and colleagues ${ }^{1}$ suggest that maternal genes have a predominant role in the risk of SPTB and that fetal genes are less important. However, a recent analysis using whole-genome-sequencing data across 816 trio families observed a substantial enrichment of de novo mutations only in preterm infants relative to term births, with the affected genes more likely to affect early fetal brain development. $^{5}$ This observation highlights the connection between SPTB and abnormal fetal development. Notably, the conclusion about the importance of maternal genes was only based on the germ-line common variants in a GWAS and requires further investigation when considering rare and de novo mutations in maternal and fetal genomes.

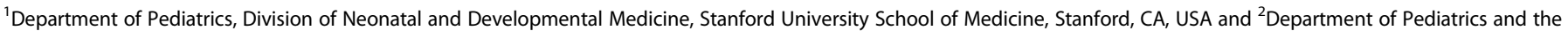
Clinical and Translational Science Institute, Tufts University School of Medicine, Boston, MA, USA

Correspondence: David K. Stevenson (dstevenson@stanford.edu)

Received: 1 November 2018 Accepted: 6 January 2019

Published online: 15 January 2019 
Several other considerations may also be relevant. Because SPTB is associated with high neonatal mortality and morbidity, genetic changes reducing the rate of SPTB would be evolutionarily favorable. This is particularly the case for the progesterone receptor (PGR), a gene playing a central role in human pregnancy and parturition. Natural selection has differentiated PGR among human populations. Recent work ${ }^{6}$ suggests that positive selection in East Asians has substantially decreased the diversity of PGR by nearly fixing evolutionarily novel alleles that likely favor the length of a normal gestation in the human species. In contrast, in the European population, the PGR locus has been mutated to a highly polymorphic state likely due to balancing selection. This might reflect local adaptation, perhaps to infectious stressors in the European environment. ${ }^{7}$ Nonetheless, these findings suggest that ancestry, which is a legacy of interactions between genes and the environment, may create genetic vulnerabilities in individuals that are exacerbated by their current environments. A variety of other social determinants could also impact a genome that was adapted for another purpose. Such determinants include sociodemographic and psychological factors that can be engaged by various physical environmental factors. Thus, we also conclude that birth timing is an evolvable trait influenced by positive selection.

Finally, a traditional GWAS may not be adequate for the identification of the many genes most likely involved in SPTB. Like many other complex human diseases, the genetic architecture of SPTB is likely polygenic; the combination of multiple genetic loci collectively conferring disease risk, with different individuals carrying different sets of mutations. Compared with the heterogeneous nature of SPTB, a GWAS might be over-simplified, where one repeatedly performs the same type of enrichment tests on each individual locus across the human genome such that the aggregated risk across multiple risk loci cannot be considered. Therefore, in addition to increasing sample size, improving existing analytic approaches to more accurately characterize the nature of a complex disease is needed. It is possible that multidimensional functional genomic data could be aggregated into a GWAS framework to boost its statistical power. ${ }^{8,9}$ It also might be more interesting and rewarding to aggregate evolutionary information into the disease interpretation framework, especially for SPTB. This is tightly associated with human reproduction and thus reflects evolutionary fitness. Such an approach would be in contrast to standard GWAS analysis, which is focused on at-risk (usually deleterious) alleles and treats each locus equally and individually. This approach might not only allow the identification of molecular innovations introduced during human evolution but also, reveal genetic components implicated in complex human diseases. Such an approach could lead to the identification of novel genes in SPTB and suggest clinically significant pathways in this condition.
In summary, we would support the conclusions of our colleagues that "development, growth, and immunity are important for pregnancy duration." We also believe that some individuals are more sensitive to SPTB triggering events than others, suggesting ancestral, as well as present gene-environment interactions. With a better understanding of such relationships, the implementation of precision health-the prediction, prevention, and curing precisely of the pathologies of pregnancy, such as SPTB - may become possible. However, a prerequisite would be the inclusion of pregnant women (and their fetuses or newborns) in comprehensive precision medicine studies, such as the All of Us Research Program of the $\mathrm{NIH}$ and other comprehensive longitudinal research studies (e.g., Project Baseline). Finally, and most importantly, efforts to address imminent SPTB should be shifted towards early detection of risk and more targeted approaches to the gene pathways involved in its initiation. Certainly, genes are relevant to a comprehensive understanding of SPTB; however, the methylome changes with age and experience, thereby making the relationship between particular genes and the various phenotypes of preterm birth somewhat elastic and definitely more complex.

\section{ADDITIONAL INFORMATION}

Competing interests: The authors declare no competing interests.

Publisher's note: Springer Nature remains neutral with regard to jurisdictional claims in published maps and institutional affiliations.

\section{REFERENCES}

1. Hallman M., et al. Spontaneous premature birth as a target of genomic research. Pediatr. Res. 2018 https://doi.org/10.1038/s41390-018-0180-z.

2. Zhang, $\mathrm{H}$. et al. A genome-wide association study of early spontaneous preterm delivery. Genet Epidemiol. 39, 217-226 (2015).

3. Huusko, J. M. et al. Whole exome sequencing reveals HSPA1L as a genetic risk factor for spontaneous preterm birth. PLoS Genet 14, e1007394 (2018).

4. Schork, N. J., Murray, S. S., Frazer, K. A. \& Topol, E. J. Common vs. rare allele hypotheses for complex diseases. Curr. Opin. Genet Dev. 19, 212-219 (2009).

5. Li, J., Oehlert, J., Snyder, M., Stevenson, D. K. \& Shaw, G. M. Fetal de novo mutations and preterm birth. PLoS Genet 13, e1006689 (2017).

6. $\mathrm{Li}$, J. et al. Natural selection has differentiated the progesterone receptor among human populations. Am. J. Hum. Genet 103, 45-57 (2018).

7. Enard, D. \& Petrov, D. A. Evidence that RNA viruses drove adaptive introgression between neanderthals and modern humans. Cell 175, 360-71 e13 (2018).

8. Lu, Q., Yao, X., Hu, Y. \& Zhao, H. GenoWAP: GWAS signal prioritization through integrated analysis of genomic functional annotation. Bioinformatics 32, 542-548 (2016).

9. Heinig, M. Using gene expression to annotate cardiovascular GWAS loci. Front Cardiovasc Med 5, 59 (2018). 